\title{
Diabetes mellitus neonatal: dos casos, diferente presentación
}

\author{
Neonatal diabetes mellitus: two cases, different presentation
}

Lorena Fernández-Ruiz,,+, Ana Belén Ariza-Jiménez, ${ }^{\ddagger}$ Carmen De la Cámara-Moraño

* Médico Interno Residente; ${ }^{\ddagger}$ Facultativo Especialista Adjunto. Unidad de Gestión Clínica de Pediatría y sus áreas específicas. Endocrinología Infantil. Hospital Universitario Reina Sofía. Córdoba, España.

\section{RESUMEN}

La diabetes mellitus neonatal (DMN) suele debutar antes de los seis meses de vida, la cual puede ser permanente o transitoria. Actualmente, se conocen más de 20 causas genéticas que condicionan su variabilidad fenotípica y su manejo terapéutico. Presentación de dos casos clínicos: El primer caso fue un recién nacido con diagnóstico de DMN transitoria, siendo como hallazgo casual la presencia de hiperglucemia. Se identificó una mutación en uno de los genes que codifican las subunidades del canal de potasio sensible a ATP $\left(\mathrm{K}_{\mathrm{ATP}}\right)$. Este paciente tuvo buena respuesta a glibenclamida. El segundo caso desarrolló una DMN transitoria secundaria a una alteración del 6q24, pero además presentó coartación de aorta, malformación de vías urinarias y cerebral. La elevación de glucemia se mantuvo bajo control con insulina. Conclusiones: Estos dos casos ilustran la variabilidad que puede presentar la DMN, su distinta evolución y manejo terapéutico, así como la importancia del papel de la genética molecular para el diagnóstico y tratamiento.

Palabras clave: Diabetes mellitus neonatal, diabetes monogénica, mutación $6 q 24$, canal de $\mathrm{K}_{\mathrm{ATP}}$, insulina, sulfanilurea.

\begin{abstract}
Neonatal diabetes mellitus (NMD) usually onset before six months of life, which can be permanent or transitory. Currently, more than 20 genetic causes are known that determine its phenotypic and treatment variability. Presentation of two clinical cases: The first case was a newborn with a diagnosis of transient DMN, in whom hyperglycemia was an accidental finding. A mutation was identified in one gene which encodes some subunities of the ATP-sensitive $K$ channel $\left(K_{\text {ATP }}\right)$. This patient had a good response to glibenclamide. The second newborn developed a transient NMD secondary to a 6q24 mutation, but this patient also presented aortic coarctation, urinary tract and brain malformations. The hyperglycemia was treated with insulin. Conclusions: These two cases illustrate the variability that can occur in DMN, both with different evolution and therapeutic management, as well as the need of molecular genetics for diagnosis and treatment.
\end{abstract}

Keywords: Neonatal diabetes mellitus, monogenic diabetes, $6 q 24$ mutation, $K_{A T P}$ channel, insulin, sulfonylurea.

\footnotetext{
+Correspondencia: LFR, lorena_fr_93@hotmail.com Conflicto de intereses: Los autores declaran que no tienen. Citar como: Fernández-Ruiz L, Ariza-Jiménez AB, De la CámaraMoraño C. Diabetes mellitus neonatal: dos casos, diferente presentación. Rev Mex Pediatr. 2020; 87(6); 232-235. https://dx.doi. org/10.35366/97686
}

\section{INTRODUCCIÓN}

La diabetes mellitus neonatal (DMN) es una forma monogénica de diabetes que suele debutar antes de los seis meses de vida, aunque puede ocurrir hasta los 12 meses. ${ }^{1}$ Actualmente, se conocen más de 20 causas genéticas de DMN que condicionan los diferentes subtipos clínicos de la enfermedad. Se estima que su incidencia varía entre 1 por 90 mil o 160 mil recién 
nacidos vivos, lo cual supone entre 1 y $4 \%$ de todas las formas de diabetes. ${ }^{2}$

Los avances de la genética molecular nos han permitido conocer su fisiopatología, ofrecer un diagnóstico prenatal y consejo genético, prever su evolución y, en muchos casos, otorgar un tratamiento personalizado. La DMN se puede clasificar en transitoria o permanente, según el curso clínico. ${ }^{3}$

En este artículo presentamos dos casos de DMN, a fin de difundir su conocimiento, ya que es una entidad poco frecuente y porque tienen distintas formas de comportamiento.

\section{CASOS CLÍNICOS}

\section{Primer caso}

Se trata de un neonato de 23 días de nacido, quien ingresó a la Unidad de Neonatología procedente de Urgencias por tumoración retroauricular sin otra sintomatología asociada. Entre sus antecedentes personales, fue un recién nacido a término de 38 semanas de gestación, con buen control prenatal. Somatometría al nacimiento: peso 3,050 g (percentil [p] 41), longitud de $52 \mathrm{~cm}$ (p 93) y perímetro cefálico de $36 \mathrm{~cm}$ (p 90).

A su ingreso, la exploración física fue normal. Estudios de laboratorio: hemograma normal, reactantes de fase aguda (RFA) negativos, glucemia de $415 \mathrm{mg} / \mathrm{dL}$, con glucosuria y sin alteraciones del equilibrio ácidobase. Se extrajeron hemocultivos y cultivos periféricos, los cuales resultaron negativos. Después, se le realizó vaciado de la tumoración con cultivo del líquido en el que creció Staphylococcus aureus. Recibió tratamiento intravenoso con cloxacilina durante siete días, sin recurrencia de la lesión.

Por la hiperglucemia, fue requerida la evaluación por Endocrinología Pediátrica, quienes identificaron abuelo por rama materna con diabetes tipo 2 , un hermano de su abuelo con diabetes tipo 1, el padre con hipercolesterolemia y la madre fumadora. Se solicitó analítica con autoinmunidad antipancreática, la cual fue negativa; los niveles de péptido $\mathrm{C}$ resultaron normales, pero HLA DRB1 y DQB1 fueron positivos. Se realizó ecografía abdominal que también resultó normal, incluyendo imagen del páncreas.

Se extrajo ADN para estudio molecular. Se inició insulina intravenosa (IV) a $0.05 \mathrm{UI} / \mathrm{kg} / \mathrm{h}$ con insulina regular. Tras ocho días y una vez logrado el control glucémico, se sustituyó la insulina IV por análogo de acción lenta (insulina detemir) vía subcutánea, 0.2 $\mathrm{UI} / \mathrm{kg} /$ día, manteniendo glucemias entre 100 у 200 $\mathrm{mg} / \mathrm{dL}$. El paciente fue dado de alta hospitalaria con este tratamiento, con la indicación de monitorización continua de glucemia.

En su evolución, requirió inicialmente un aumento de la insulina detemir a $0.3 \mathrm{UI} / \mathrm{kg} /$ día repartida en dos dosis, mostrando buena ganancia ponderoestatural y controles glucémicos en alrededor a $200 \mathrm{mg} / \mathrm{dL}$ durante el día. En el estudio molecular, se identificó una mutación patogénica c.3544C > T p.(Arg1182Trp) en heterocigosis del gen $A B C C 8$, que está asociada con la DMN permanente o transitoria, la cual puede ser heredada o de novo.

Eventualmente, al mostrar buen control glucémico se inició tratamiento con sulfonilureas (SU): glibenclamida $0.2 \mathrm{mg} / \mathrm{kg} /$ día repartida en dos dosis, con disminución progresiva de insulina. En la actualidad se encuentra sin tratamiento ya que se pudo retirar también la glibenclamida.

\section{Segundo caso}

Ingresó a la Unidad de Neonatología un neonato de 16 días de vida, procedente de la Unidad Pediátrica de Cuidados Intensivos tras una cirugía de coartación de aorta, realizada a los ocho días de vida. Entre sus antecedentes, recién nacido a término de 37 semanas de gestación con diagnóstico prenatal de coartación de aorta y riñón izquierdo poliquístico. Somatometría al nacimiento: peso 2,030 g $(<\mathrm{p} \mathrm{3),} \mathrm{longitud} 45 \mathrm{~cm}(\mathrm{p} \mathrm{4})$ y perímetro cefálico $32 \mathrm{~cm} \mathrm{(p} \mathrm{20).}$

$\mathrm{Al}$ ingreso se determinó glucosa de $258 \mathrm{mg} / \mathrm{dL}$, urea de $25 \mathrm{mg} / \mathrm{dL}$, creatinina de $0.66 \mathrm{mg} / \mathrm{dL}$, GGT (gammaglutamil transferasa) de 1,303 U/L, hemograma y reactantes de fase aguda normales. Cultivos periféricos resultando negativos. En la bioquímica de orina, hiperuricosuria, glucosuria y proteinuria tubular.

Debido a que la hiperglucemia fue persistente, se estudió por Endocrinología Pediátrica. No se identificaron antecedentes familiares de autoinmunidad o enfermedades endocrinas. Péptido $\mathrm{C}$ y hormonas tiroideas normales, anticuerpos antipancreáticos negativos. Se solicitó estudio genético mediante arreglo de hibridación genómica comparativa (CGH array).

Se inició perfusión continua de insulina IV, con requerimientos de 0.02 a $0.03 \mathrm{U} / \mathrm{kg} / \mathrm{hora}$. Tras lograr un buen control glucémico, la insulina IV se sustituyó por análogo de acción lenta, glargina, por vía subcutánea cada 24 horas; sin embargo, fue necesario cambiar a infusión subcutánea continua de insulina (ISCI) con dosis máxima de $1.2 \mathrm{UI} / \mathrm{kg} /$ día hasta tener una pro- 
gresiva normalización de la glucemia. La insulina se retiró una semana previa al alta.

Debido a la elevación de GGT, se realizó ecografía hepatopancreática que fue normal. Se inició tratamiento con ácido ursodesoxicólico, con progresiva normalización de los valores de GGT.

Por otro lado, en electroencefalograma se determinó la presencia de crisis occipitales bilaterales pero con ausencia de crisis clínicas, por lo que se inició tratamiento con fenobarbital. En la resonancia magnética se observó cavum del septum pellucidum y aumento de espacios extracerebrales, sin otros hallazgos.

El CGH array demostró una ganancia de 1.94 megabases $(\mathrm{Mb})$ en la citobanda 6q24.2-6q24.3 de una región que se asocia con $\mathrm{DMN}$, la cual se interpretó como variante de significado incierto con probabilidad de ser patogénica. También, se detectaron dos reordenamientos contiguos en la citobanda 20p13: una deleción terminal de $1.41 \mathrm{Mb}$ y una duplicación intersticial de $2.39 \mathrm{Mb}$. Ambas se interpretaron como patogénicas. Ante tal resultado, se ofreció realizarles estudio genético también a los progenitores, siendo rechazado por el padre. El estudio de segregación en la madre mostró que ninguna de las variantes detectadas en su hijo fue heredadas por vía materna.

Tras el alta, nuestro paciente presentó una evolución favorable, mantenido niveles normales de glucemia sin requerir insulina o sulfonilureas.

\section{DISCUSIÓN}

La DMN no siempre está presente durante el periodo neonatal inmediato, pudiéndose diagnosticar hasta los 12 meses postnatales. ${ }^{1}$ Para el diagnóstico, se deben tener en cuenta otras situaciones que provocan hiperglucemia neonatal, tales como sepsis, prematuridad, nutrición parenteral, fármacos (como los esteroides), y situaciones de estrés, que incrementan los niveles de hormonas contrarreguladoras. ${ }^{4}$ Los pacientes pueden debutar insidiosamente con polidipsia, poliuria y fallo de medro; de forma aguda, con cetoacidosis o alteración del estado mental, o accidentalmente, con bajo peso al nacer y hallazgo casual de hiperglucemia durante el periodo neonatal, como fue el caso de nuestros pacientes. ${ }^{5}$

Ante sospecha de DMN, la evaluación inicial debe incluir: glucemia, cetonemia, cetonuria, gasometría y péptido $\mathrm{C}$; además de hemograma con RFA como parte del diagnóstico diferencial. Además de ecografía pancreática, puesto que la presencia o ausencia de alteraciones en este órgano tienen consecuencias terapéuticas. ${ }^{6}$
El tratamiento inicial en el manejo de la hiperglucemia neonatal siempre es con insulina IV; sin embargo, el pronóstico varía en caso de que su origen sea genético. De ahí la importancia de solicitar un estudio genético, el cual se recomienda solicitar con glucemias $>250 \mathrm{mg} / \mathrm{dL}$ durante siete a 10 días, o inmediatamente en caso de que el paciente presente hiperglucemias extremas. ${ }^{6}$

Pese al hallazgo accidental y transitorio de hiperglucemia de los casos presentados, ambos son muy distintos en cuanto a su origen genético, contexto clínico y manejo terapéutico. En el primer caso, nuestro paciente presentó una mutación del canal de potasio sensible a ATP $\left(\mathrm{K}_{\mathrm{ATP}}\right)$. Los canales $\mathrm{K}_{\mathrm{ATP}}$ son complejos heteroctaméricos constituidos por cuatro subunidades Kir6.2, que son formadoras de poros y cuatro subunidades regulatorias SUR1 codificadas por los genes KCNJ11 y ABCC8, respectivamente. ${ }^{3,7,8}$ Estas subunidades regulan la secreción de insulina. Las mutaciones en estos genes evitan el cierre de los canales $\mathrm{K}_{\mathrm{ATP}}$, haciendo que la célula no se despolarice y, por tanto, no libere insulina en respuesta a la hiperglucemia. Cabe señalar que éstos son la causa más frecuente de DMN permanente y la segunda de DMN transitoria. ${ }^{7,9}$

Cuando la variante patogénica es $A B C C 8$, hasta en $66 \%$ se presenta como DMN transitoria y el peso de nacimiento no se ve tan afectado, ${ }^{7}$ tal como ocurrió con nuestro paciente. La peculiaridad terapéutica de este tipo de DMN es su respuesta de sulfonilureas (SU), que de 80 a $90 \%$ de los casos permite suspender insulina, tal como sucedió en nuestro caso, siendo la glibenclamida el fármaco de elección. ${ }^{6,10}$ Las SU se unen a SUR1 y cierran el $\mathrm{K}_{\mathrm{ATP}}$, lo que permite que se despolarice la membrana y se restablezca la funcionalidad de la célula beta. ${ }^{10}$ Así, en el primer caso, la SU fue suficiente para sustituir la terapia insulínica, pero finalmente también se suspendió dado el carácter transitorio que mostró la enfermedad.

El segundo paciente presentó una mutación en el locus 6q24, dando lugar a la DMN transitoria. Las alteraciones en el brazo largo del cromosoma 6 suponen hasta dos tercios de todas las formas de DMN. ${ }^{9} \mathrm{En}$ condiciones normales, la región 6q24 del cromosoma materno se silencia por una metilación de sus bases; esta mutación produce una hipometilación a dicho nivel, que conlleva una sobreexpresión de dos genes: PLAGL1 e HYMAI, dando lugar a la enfermedad. ${ }^{11,12}$ Las tres causas de sobreexpresión de los genes de esta región descritas son: duplicación paterna no balanceada del 6q24; disomía uniparental paterna del cromoso- 
ma 6; o bien, hipometilación materna del $6 q 24 .{ }^{12}$ En nuestro caso, se pudo descartar la herencia materna de las variantes patogénicas, pero no se pudo concluir el mecanismo paterno de sobreexpresión.

Por otro lado, también se han descrito malformaciones congénitas en pacientes con DMN asociadas con el locus 6q24, como macroglosia y hernia umbilical. ${ }^{13}$ Otras anomalías menos frecuentes son: dismorfia facial, malformaciones del tracto urinario (duplicación renal, hidronefrosis y ectasia piélica), cardiacas (ductus arterioso persistente, tetralogía de Fallot, foramen oval persistente y defectos auriculares) así como en dedos y uñas; además de hipotiroidismo. ${ }^{13,14}$ Nuestro paciente presentó coartación de aorta, displasia renal multiquística izquierda y malformación cerebral.

Los pacientes con mutación del 6q24 desarrollan hiperglucemia no cetósica en el periodo neonatal, pero parece ser que la hiperglucemia se presenta nuevamente durante la adolescencia, persistiendo en la edad adulta. $13,15,16$

\section{REFERENCIAS}

1. Rubio-Cabezas O, Ellard S. Diabetes mellitus in neonates and infants: genetic heterogeneity, clinical approach to diagnosis, and therapeutic options. Horm Res Paediatr. 2013; 80(3): 137-146.

2. Letourneau LR, Carmody D, Wroblewski K, Denson AM, Sanyoura $\mathrm{M}$, Naylor RN et al. Diabetes presentation in infancy: high risk of diabetic ketoacidosis. Diabetes Care. 2017; 40(10): e147-e148.

3. Gálvez-López AG, Villalobos-Lizardi JC, Águila-Cano R. Diabetes neonatal monogénica. Rev Med MD. 2018; 10(1): 29-34.

4. Hay WW, Rozance PJ. Neonatal hyperglycemia: causes, treatments, and cautions. J Pediatr. 2018; 200: 6-8.
5. Karges B, Meissner T, Icks A, Kapellen T, Holl RW. Management of diabetes mellitus in infants. Nat Rev Endocrinol. 2012; 8: 201-211.

6. Lemelman MB, Letourneau L, Greeley SAW. Neonatal diabetes mellitus: an update on diagnosis and management. Clin Perinatol. 2018; 45(1): 41-59.

7. Babenko AP, Polak M, Cavé $H$, Busiah $K$, Czernichow $P$, Scharfmann R et al. Activating mutations in the ABBC8 gene in neonatal diabetes mellitus. N Engl J Med. 2006; 355: 456-466.

8. McTaggart JS, Clark RH, Ashcroft FM. The role of the KATP channel in glucose homeostasis in health and disease: more than meets the islet. J Physiol. 2010; 588(17): 3201-3209.

9. De Franco E, Flanagan SE, Houghton JAL, Allen HL, MacKay DJG, Temple IK et al. The effect of early, comprehensive genomic testing on clinical care in neonatal diabetes: an international cohort study. Lancet. 2015; 386(9997): 957-963.

10. Rafiq M, Flanagan SE, Patch AM, Shields BM, Ellard S, Hattersley AT et al. Effective treatment with oral sulfonylureas in patients with diabetes due to sulfonylurea receptor 1 (SUR1) mutations. Diabetes Care. 2008; 31(2): 204-209.

11. Mackay DJG, Boonen SE, Clayton-Smith J, Goodship J, Hahnemann JMD, Kant SG et al. A maternal hypomethylation syndrome presenting as transient neonatal diabetes mellitus. Hum Genet. 2006; 120: 262-269.

12. Polak M, Cavé H. Neonatal diabetes mellitus: a disease linked to multiple mechanisms. Orphanet J Rare Dis. 2007; 2(1): 1-11.

13. Docherty LE, Kabwama S, Lehmann A, Hawke E, Harrison L, Flanagan SE et al. Clinical presentation of 6q24 transient neonatal diabetes mellitus (6q24 TNDM) and genotype-phenotype correlation in an international cohort of patients. Diabetologia. 2013; 56(4): 758-762.

14. Gore RH, Nikita ME, Newton PG, Carter RG, Reyes-Bautista J, Greene CL. Duplication 6q24: more than just diabetes. J Endocr Soc. 2020; 4(5): 6-9.

15. Ferraro M, Ramos O, Asenjo S, García H, Lema A, Blanco M et al. DN ¿Transitoria o definitiva? Asoc Latinoam Diabetes. 2007; 15(2): 55-59.

16. Jiménez-Uscanga RD, Ordóñez-Gutiérrez E, Jiménez-Urueta $P$, Gómez-Guillermoprieto L. Diabetes mellitus neonatal. Seguimiento a largo plazo de un paciente. Acta Pediatr Mex. 2010; 31(6): 274-280. 